\title{
Machine Learning-based CT Radiomics Model Distinguishes COVID-19 From Non-COVID-19 Pneumonia
}

\author{
Hui Juan Chen \\ Hainan General Hospital \\ Li Mao \\ Deepwise Inc. \\ Yang Chen \\ Hainan General Hospital \\ Li Yuan \\ Hainan General Hospital \\ Fei Wang \\ Hainan General Hospital \\ Xiuli Li \\ Deepwise Inc. \\ Qinlei Cai \\ Hainan General Hospital \\ Jie Qiu \\ Hainan General Hospital \\ Jie Tian \\ Chinese Academy of Science
}

Feng Chen ( $\nabla$ fenger0802@163.com)

Department of Radiology, Hainan General Hospital (Hainan Affiliated Hospital of Hainan Medical University), NO. 19, XIUHUA ST, XIUYING DIC, Haikou, Hainan, 570311, P.R. China.

\section{Research Article}

Keywords: Machine learning, radiomics, Coronavirus Disease 2019 (COVID-19), non-COVID-19 pneumonia

Posted Date: January 14th, 2021

DOI: https://doi.org/10.21203/rs.3.rs-143801/v1

License: (c) (i) This work is licensed under a Creative Commons Attribution 4.0 International License. Read Full License 


\section{Abstract}

Background: To develop a machine learning-based CT radiomics model is critical for the accurate diagnosis of the rapid spread Coronavirus disease 2019 (COVID-19).

Methods: In this retrospective study, a total of 326 chest CT exams from 134 patients (63 confirmed COVID-19 patients and 71 non-COVID-19 patients) were collected from January 20 to February 8, 2020. A semi-automatic segmentation procedure was used to delineate the region of interest (ROI), and the radiomic features were extracted. The Support Vector Machine(SVM) model was built on the combination of the 4 groups of features, including radiomic features, traditional radiological features, quantifying features and clinical features, by repeated cross-validation procedure and the performance on the time-independent testing cohort was evaluated by the area under the receiver operating characteristic curve (AUC), accuracy, sensitivity and specificity.

Results: For the SVM model that built on the combination of 4 groups of features(integrated model), the per-exam AUC of $0.925(95 \%$ Cl: 0.856 to 0.994) was reached for differentiating COVID-19 on the testing cohort, and the sensitivity and specificity were $0.816(95 \%$ Cl: 0.651 to 0.917$)$ and 0.923(95\% Cl: 0.621 to 0.996), respectively. For the SVM models that built on radiomic features, radiological features, quantifying features and clinical features individually, the AUC on the testing cohort reached $0.765,0.818,0.607$ and 0.739 respectively, significantly lower than the integrated model, except for the radiomic model.

Conclusion: The machine learning-based CT radiomics models may accurately detect COVID-19, helping clinicians and radiologists to identify COVID-19 positive cases.

\section{Introduction}

The Coronavirus Disease 2019 (COVID-19) has widely and rapidly spread throughout the world since late December 2019(1, 2). The newly emerging disease is highly contagious and may cause severe acute respiratory distress or multiple organ failure in severe cases (3-6). The World Health Organization (WHO) declared the outbreak of COVID-19 as a "public health emergency of international concern" (PHEIC) on January 30, 2020.

At present, the gold standard for the diagnosis of COVID-19 is reverse-transcription polymerase chain reaction (RT-PCR). However, a high false negative rate (7) and the shortage of RT-PCR assay in the early stage of the outbreak limited the early detection and treatment of the presumptive patients $(8,9)$. This speeded up the spread of COVID-19. Therefore, fast diagnosis is important for controlling the spread of COVID-19. Recent studies have demonstrated that computed tomography (CT), as a non-invasive imaging approach, is of great value in detecting lung lesions in patients with COVID-19 infection $(2,10)$. Besides, CT had much higher sensitivity than initial RT-PCR in diagnosing COVID-19 (8, 9). Consequently, CT could be used as an effective tool for early detection and diagnosis of COVID-19. We should not neglect the fact that COVID-19 may have certain similar CT imaging features with other types of pneumonia, thus making it hard to differentiate. Although measures have been taken to control the spread of the disease, there have been 23,518,343 confirmed cases of COVID-19 globally, as of 3:49pm CEST, 25 August 2020, including 810,492 deaths. Concerning the pandemic of COVID-19, accurate and fast diagnosis of COVID-19 is vital to isolate infected patients and slow down the spread of this disease.

Current studies have demonstrated that artificial intelligence could distinguish COVID-19 from other pneumonia (11, 12), improve radiologists' performance in distinguishing COVID-19 from non-COVID-19 pneumonia on chest CT and provide clinical prognosis with good accuracy that can assist clinicians to timely adjust their clinical management and allocate resources appropriately(13-19). However, CT manifestations of COVID-19 resemble to other types of pneumonia. Additionally, the non-COVID-19 diseases included as a comparison group are long before the COVID-19 outbreak. Since the CT manifestations of common pneumonia resemble to that of COVID-19 pneumonia. The most difficult situation in clinical diagnosis and treatment is to identify other types of pneumonia that occurred in the same period as the epidemic of COVID-19.

In recent years, much attentions had been paid to radiomics in disease diagnosis and treatment outcome evaluation (20, 21). Specifically, radiomics is of great value in medical imaging because of its ability to extract high throughput of quantitative descriptors from routine computed tomography (CT) studies(21). Radiomics was applied to many fields of cancer, such as tumor detection, preoperative prediction of lymph node metastasis and therapeutic response assessment $(20,22,23)$. Recently, radiomics have been proved to be helpful in COVID-19 screening, diagnosis, prediction of hospital stay, assessing the imaging characteristics and risk factors associated with adverse composite endpoints in patients with COVID-19 pneumonia (24-27). However, these studies were limited in small sample size. In the study of Qi et al., 31 patients were finally included in the study(25). Some did not extract high-throughput imaging features(27). Besides, no radiomics study of COVID-19 has been done compared with non-COVID-19 pneumonia of the corresponding period, which is difficult to differentiate from COVID-19. The purpose of this study was to develop and test machine learning-based CT radiomics models for the detection of COVID-19. Other types of pneumonia exams were also included to test the robustness of the model.

\section{Materials And Methods}




\section{Study population}

This retrospective study was waived by the ethics committees of the Hainan General hospital. In total, 74 patients confirmed with COVID-19 infection from January 20 to February 8, 2020, and 82 patients with other types of pneumonia of the corresponding period were collected. In the COVID-19 dataset, 63 patients were finally included who met the following inclusion criteria: (i) RT-PCR confirmed COVID-19; (ii) non-contrast CT at diagnosis time; (iii) positive CT findings. 71 patients with Non-COVID-19 pneumonias were included who met the following inclusion criteria: (i) RTPCR excluded COVID-19; (ii) non-contrast CT at diagnosis time; (iii) pneumonia highly suspected with COVID-19 at CT. The exclusion criteria were as follows: 1) contrast CT exam; 2) exams without slice thickness of $1 \mathrm{~mm}$; 3 ) negative CT findings. Finally, 326 chest CT exams from 134 patients were included in this study (Fig. 1). The average age is $47.0 \pm 15.4$ years. Finally, we included 244 (75\%) exams for COVID-19 and $82(25 \%)$ for nonCOVID-19 pneumonia in the study.

All the COVID-19 were confirmed as positive by RT PCR and were acquired from January 21, 2020, to Feb 8, 2020. The most common symptoms were fever (82\%) and cough (77\%). Each patient had one or multiple CT scans during the progression of the disease. The follow-up study was performed until February 19,2020.

Other types of pneumonia patients were selected from the hospital of the corresponding period between January 23 to March 16,2020 . For 82 patients with negative RT-PCR results, pneumonia was diagnosed according to the Infectious Diseases Society of America/American Thoracic Society (IDSA/ATS) guidelines (28). Patients with at least one of the following clinical symptoms including cough, sputum, fever, dyspnea, and pleuritic chest pain, plus at least one finding of coarse crackles on auscultation or elevated inflammatory biomarkers, in addition to a new pulmonary infiltration on chest CT, would be diagnosed to be infected with pneumonia. The admission distribution of the patients with other types of pneumonia was: outpatient $(86 \%, 61$ of 71$)$, inpatient $(14 \%, 10$ of 71$)$. No one received laboratory confirmation of the etiology because of limited medical resources.

CT examinations were performed on the NeuViz 128 CT (Neusoft, China) with automatic tube current (300 mA-496 mA), tube voltage $=120 \mathrm{kV}$. The pitch was set at 1.5 and breath-hold at full inspiration. The slice-thickness of each CT scan was $1 \mathrm{~mm}$. The reconstruction matrix was $512 \times 512$ pixels.

All the CT scans were split with a ratio of 85:15 into a training cohort and a testing cohort at the patient level according to the visiting time of the hospital. The features selection and model building were performed on the training cohort, and the testing cohort was not used for the training procedure.

All subjects' demographic characteristics and clinical data were retrospectively reviewed and collected, including age, gender, exposure history, diabetes, hypertension, chronic obstructive pulmonary disease(COPD), chronic liver disease, chronic kidney disease, cancer, cardiovasular disease, fever, cough, myalgia, fatigue, headache, nausea, diarrhea, bellyache, dyspnea, other symptoms, white blood cell count, number of neutrophils, lymphocyte count, hemoglobin and platelet count. The patient demographic statistics are summarized in Table 1. Imaging Protocol

The flow chart of data collection, ROI and features annotation, radiomics and quantity feature extraction, model building and evaluation are shown in Fig. 2.

\section{Lesion segmentation and radiological evaluation}

The pneumonia lesions were segmented semi-automatically. Firstly, the anonymized thin-slice DICOM format non-enhanced CT images were imported into an Al pneumonia assessment system, on which the pneumonia lesions were automatically detected and delineated. On the assessment platform, a MVP-Net (Multi-View FPN with Position-aware attention) which was trained on the NIH DeepLesion dataset and achieved state-of-the-art performance(29), was used to detected the abnormal pattern and classified them into consolidation and ground-glass opacity. Then a 2D U-Net model that trained on a local dataset of about 8,000 lung CT images was used to segment detected consolidation and groundglass opacity lesions. Besides, pulmonary lobes were segmented by a pre-trained lobe segmentation model(30). Secondly, fifteen radiologists with more than 5 years of experience in chest imaging, blind to the knowledge of the pathological report and other clinical information, refined the segmentation result (Region of Interest, ROI) and evaluated the radiological characteristics. Each series was refined and evaluated by one of the fifteen radiologists. The segmentations and radiological characteristics were confirmed by two radiologists (F. C and Y.C) with 16 and more than 30 years of experience.

The 7 radiological characteristics included ground glass opacity, crazy paving pattern, halo sign, reversed halo sign, vascular perforating in the lesion, subpleural line and lesion locations (Fig. 3). For each series, the frequency of the radiological characteristics occurring was used for modeling.

\section{Quantifying CT characteristics and radiomics features}

The segmentation results were used to extract quantifying CT characteristics and radiomics features. 
There were a total of 33 quantitative characteristics. Beside of the segmentation results, the Al pneumonia assessment system also provided the number of lesions that suffered bulla, emphysema, pleural thickening, reticular, and stripe, which were included as quantitative characteristics. From the segmentations, the mean and standard deviation of the CT values of the consolidation lesions, ground glass lesions and both of them were included. Then the volumes of the consolidation lesions, ground glass lesions and their sum were calculated. From the volumes, some ratios were calculated, including the volumes of the consolidation lesions versus the volumes of the entire pulmonary and the five pulmonary lobes respectively, the ground glass lesions versus the volumes of the entire pulmonary and the five pulmonary lobes respectively and the volumes of both type of lesions versus the volumes of the entire pulmonary and the five pulmonary lobes respectively.

Before the radiomics features were extracted, the pixel spacing of images was resampled to $1.0 \mathrm{~mm}$ per pixel by the BSpline algorithm. Besides the original images, the wavelet filters or Laplacian of Gaussian filters were performed to generate several filtered images. A total of 1218 radiomics features were extracted from the manual confirmed ROIs of the original images and the filtered images by PyRadiomics V2.1.0, including (1) 252 First-order features; (2) 14 Shape-based features; (3) 308 Gray Level Co-occurrence Matrix Features (GLCM); (4) 224 Gray Level Size Zone Matrix Features (GLSZM); (5) 224 Gray Level Run Length Matrix Features (GLRLM); (6) 196 Gray Level Dependence Matrix Features (GLD-ZM). The preprocessing methods and radiomic feature descriptions are detailed in Supplementary Information 1.1. and 1.2.

\section{Development of predictive models}

There were 4 groups of features that enrolled in model building: radiomics features, radiological features, quantity features and clinical features. The Support Vector Machine(SVM) model with radial basis function kernel were built on the 4 groups of features individually and on the combination of them.

Before model building, all numerical features were normalized by z-score method, and the categorical features were encoded by one-hot encoder. To avoid overfitting, the feature selection methods were used to reduce the number of features. The optimal parameters of the combination of the feature selection method and the model were found by grid search using a ten-run 5 -fold cross validation procedure on the training cohort. After the optimal params were determined, the entire training cohort was used to build the model and the performance on the testing cohort was evaluated. After the cross-validation procedure, the threshold that maximum the Youden Index on the validation cohort was used to cut-off the discriminative score to differentiate the COVID-19 from other pneumonia.

The features were selected by a two-step method. (1)The Mann-Whitney U test was used and the p values were correct by Benjamini-Hochberg method. The feature that significantly different $(p<0.05)$ between the COVID-19 cohort and non-COVID-19 cohort was preserved. $(2)$ the minimumredundancy maximum-relevancy (mRMR) method was used and the number of selected features was determined by the cross-validation procedure. Specially, for the radiological features, the mRMR procedure was removed because there were only 7 radiological features.

The discrimination performance of the model was evaluated by the area under the receiver operator characteristic curve (AUC), accuracy (ACC), sensitivity and specificity. The AUCs of the SVM model that built on the combined features and that on each individual feature group were compared by using the Delong test. Because the SVM model with radial basis function kernel is nonlinear, the feature importance cannot be derived directly. The permutation importance(31) was used to evaluate the feature importance and the AUC was used to measure the difference between the baseline and the model that built with permutated feature.

\section{Results}

\section{Clinical data}

Table 1 demonstrates the study population characteristics for the training and testing cohorts. Data related to age, exposure history, cough, myalgia, fatigue, headache and diarrhea were significantly different between COVID-19 and other types of pneumonia in the training cohort ( $p<$ 0.05). Regarding the laboratory results, the white blood cell count, number of neutrophils were significantly lower in the COVID-19 group than that in the negative group $(\mathrm{p}<0.05)$ for both training cohort and testing cohort. In addition, the lymphocyte and plate count were significantly lower in the COVID-19 group than in the other types of pneumonia group $(p<0.05)$.

\section{Evaluation of the model performance}

A total of 1128 radiomic features were extracted from each patient, the correlation cluster map was shown in Fig. 4.

For the SVM model that built on the combination of 4 groups of features, it reached an AUC of 0.984(0.971 to 0.997), 0.893(0.841 to 0.946), and $0.925(0.856$ to 0.994$)$ on the training, cross-validation and testing cohort. For the sensitivity and specificity, it reached 0.816 and 0.923 on the test cohort. For the SVM models that built on radiomic features, radiological features, quantifying features and clinical features individually, the AUC on the testing cohort reached $0.765(95 \% \mathrm{Cl}: 0.585$ to 0.946$), 0.818(95 \% \mathrm{Cl}: 0.698$ to 0.938$), 0.607(95 \% \mathrm{Cl}: 0.414$ to 0.8$)$ and $0.739(95 \% \mathrm{Cl}$ : 0.58 to 0.898) respectively, significantly lower than the integrated model, except for the radiomic model. The details of the performance are shown in table. 2 and the ROC curve of the 4 SVM models on the time-independent test cohort was shown in Fig. $\mathbf{5 .}$ 
There were 30 features that involved in the integrated SVM model building, included 14 radiomic features, 9 clinical features, 4 quantifying features, and 3 radiological features. The feature importance of these features was shown in Fig. 6.

Fig. 7 showed the decision function value distribution of the non-COVID-19 pneumonia and COVID-19 in the test cohort. The function values are proportional to the distance of the patient to the separating hyperplane, thus indicate the model's confidence in the result of classification. The separating hyperplane was adjusted to maximize the Youden index on the cross-validation cohort. From the CT images, we could see that when the lesions of COVID-19 were at absorption stage, it became small and thus it is difficult to differentiate from non-COVID-19 pneumonia. On the contrary, when the lesions of COVID-19 were relatively big, it is easy to differentia it from non-COVID-19 pneumonia with typical lesion locations and CT manifestation.

\section{Discussion}

In this study, we developed and tested a machine learning-based CT radiomics model for detecting COVID-19 from chest CT images. CT radiomics features of lesions were extracted, and machine-learning model showed good performance for training, inter-validation and testing cohort. On the testing dataset, our result revealed that this model achieved high sensitivity $0.816(95 \% \mathrm{Cl}: 0.651$ to 0.917$)$ and high specificity of $0.923(95 \% \mathrm{Cl}$ : 0.621 to 0.996$)$ in diagnosing COVID-19.

Since the outbreak of COVID-19, clinical characteristics have been regarded as important clues for diagnosing COVID-19. However, the exact role of clinical characteristics in the diagnosis of COVID has not yet been evaluated. Our present study revealed that clinical features were valuable, but not the only strong predictor for diagnosing COVID-19. This result is of great important, since a number of cases is still rising all over the world. We have included both COVID-19 patients without history of exposure and non-COVID-19 patients with history of exposure in the current study. Exposure history has been regarded as an important role in diagnosing COVID. Besides, our study demonstrated that when compared with nonCOVID-19 patients, COVID-19 patients had significantly lower leukocyte counts, neutrophils, lymphocyte and platelet count. This could be explained by that because COVID-19 belongs to viral infection, whereas non-COVID-19 patients were likely diagnosed as bacterial infection with high leukocyte count. This is consistent with previous study that normal or abnormally low leukocyte count and lymphocyte was found to be a significant indicator for diagnosing COVID-19(32).

CT manifestations of COVID-19 have been deemed as an indispensable role for the clinical diagnosis of COVID-19 (33). However, few studies have elucidated the role of CT features in diagnosing COVID-19. Therefore, we have assessed the diagnostic value of radiological characteristics including ground glass opacity, crazy paving pattern, halo sign, reversed halo sign, vascular perforating in the lesion, subpleural line and lesion locations in our study. We found that when radiological features were included alone, the model revealed the good performance of AUCs for training, validation and testing cohort, $92.2 \%, 86.9 \%$ and $81.8 \%$, respectively. Our study indicated that CT is valuable for diagnosing COVID-19.

The encouraging diagnostic performance of the machine learning-based CT radiomics model indicates that radiomics may be particularly helpful for the detection of COVID-19 as the AUC of other models in the testing dataset were significantly lower than the integrated model, except for the radiomics model. Radiomics features in our model included first order features, shape-based features and the distribution, correlation and variance in gray level intensities. These radiomics features described the relationship between voxels and contained quantitative information on the spatial heterogeneity of pneumonia lesions. Importantly, when radiomics features were included alone, the model revealed the good performance of AUCs for training, validation and testing cohort, $96.2 \%, 82.8 \%$ and $76.5 \%$, respectively. By combining the radiological features, quantifying features and clinical characteristics, the performance of the model was significantly improved. The model demonstrated satisfied AUCs more than $89 \%$ on training, validation and testing cohorts, indicating that the models have the potential to be applied in a general situation. By using deep learning techniques, previous study was able to distinguish COVID-19 from community acquired pneumonia(11). We were able to collect a number of patients with other types of pneumonia diagnosis on CT of the corresponding period. More importantly, these pneumonias were highly suspected of COVID-19 in consideration of the epidemic, CT findings, and laboratory results.

A majority of the countries all over the world have been affected by COVID-19. Early diagnosis is of importance for preventing the spread of the disease. Though RT-PCR is considered as the gold standard for the diagnosis of COVID-19, CT is used as an effective supplementary tool for the diagnosis of COVID-19 $(8,9)$. Our study revealed that the machine learning-based CT radiomics model by combining radiomics, subjective characteristics, quantitative characteristics and clinical characteristics achieved good performance for the diagnosis of COVID-19 and differentiating it from non-COVID-19 pneumonia.

The study has several limitations. First, the sample size was relatively small. A larger prospective multicenter cohort is needed to test the effectiveness of machine learning-based CT radiomics models. Second, patients with non-COVID-19pneumonia did not receive laboratory confirmation of the etiology because of limited medical resources during the COVID-19 outbreak. Thirdly, we did not use quantitative characteristics to evaluate the evolution of the disease. Future work should include quantitative information regarding disease progression.

\section{Conclusions}


In conclusion, a machine learning-based CT radiomics model is valuable for accurately detecting COVID-19, which would be helpful for clinicians and radiologist to identify COVID-19 patients.

\section{Abbreviations}

COVID-19: Coronavirus disease 2019; ROI: region of interest; SVM: Support Vector Machine; WHO: World Health Organization; PHEIC: public health emergency of international concern; RT-PCR: reverse-transcription polymerase chain reaction; CT: computed tomography; GLSZM: Gray Level Size Zone Matrix Features; GLRLM: Gray Level Run Length Matrix Features; GLD-ZM: Gray Level Dependence Matrix Features; AUC: area under the receiver operator characteristic curve.

\section{Declarations}

\section{Ethical Approval and Consent to participate}

The authors are accountable for all aspects of the work in ensuring that questions related to the accuracy or integrity of any part of the work are appropriately investigated and resolved. The study was conducted in line with the principles of the Declaration of Helsinki, and Institutional Review Board approval has been obtained. The written informed consent for this retrospective study was waived.

\section{Consent for publication}

Not applicable.

\section{Availability of supporting data}

The datasets used during the current study are available from the corresponding author on reasonable request.

\section{Competing interests}

The authors have no conflicts of interest to declare.

\section{Funding}

This work was supported by the Key R \& D plan of Hainan Province (ZDYF (XGFY) 2020001); National Nature Science Foundation of China [grant number 81971602, 81760308, 81801684]; the Program of Hainan Association for Science and Technology Plans to Youth R \& D Innovation [QCXM201919]; Hainan Provincial Natural Science Foundation of China [818MS124].

\section{Authors' contributions}

JT and FC conceived of and designed the study. HJC, LM, YC, LY, FW, XL, QC and FC collected and assembled the data: HJC, LM, YC, LY, FW, XL, QC and JQ contributed to the data analysis and interpretation. All authors contributed to the manuscript writing and final approval of manuscript.

\section{Acknowledgements}

We thank all patients for their participation.

\section{Authors' information}

Jie Tian, Email: jie.tian@ia.ac.cn.

Feng Chen, Email: fenger0802@163.com.

\section{References}

1. Wang D, Hu B, Hu C, Zhu F, Liu X, Zhang J, et al. Clinical Characteristics of 138 Hospitalized Patients With 2019 Novel Coronavirus-Infected Pneumonia in Wuhan, China. Jama. 2020 Feb 7.

2. Huang C, Wang Y, Li X, Ren L, Zhao J, Hu Y, et al. Clinical features of patients infected with 2019 novel coronavirus in Wuhan, China. Lancet (London, England). 2020 Jan 24;395(10223):497-506.

3. Chen N, Zhou M, Dong X, Qu J, Gong F, Han Y, et al. Epidemiological and clinical characteristics of 99 cases of 2019 novel coronavirus pneumonia in Wuhan, China: a descriptive study. Lancet (London, England). 2020 Feb 15;395(10223):507-13.

4. Phan LT, Nguyen TV, Luong QC, Nguyen TV, Nguyen HT, Le HQ, et al. Importation and Human-to-Human Transmission of a Novel Coronavirus in Vietnam. The New England journal of medicine. 2020 Feb 27;382(9):872-4. 
5. Liu YC, Liao CH, Chang CF, Chou CC, Lin YR. A Locally Transmitted Case of SARS-CoV-2 Infection in Taiwan. The New England journal of medicine. 2020 Mar 12;382(11):1070-2.

6. Li Q, Guan X, Wu P, Wang X, Zhou L, Tong Y, et al. Early Transmission Dynamics in Wuhan, China, of Novel Coronavirus-Infected Pneumonia. The New England journal of medicine. 2020 Jan 29.

7. Chan JF, Yuan S, Kok KH, To KK, Chu H, Yang J, et al. A familial cluster of pneumonia associated with the 2019 novel coronavirus indicating person-to-person transmission: a study of a family cluster. Lancet (London, England). 2020 Jan 24.

8. Ai T, Yang Z, Hou H, Zhan C, Chen C, Lv W, et al. Correlation of Chest CT and RT-PCR Testing in Coronavirus Disease 2019 (COVID-19) in China: A Report of 1014 Cases. Radiology. 2020 Feb 26:200642.

9. Fang Y, Zhang H, Xie J, Lin M, Ying L, Pang P, et al. Sensitivity of Chest CT for COVID-19: Comparison to RT-PCR. Radiology. 2020 Feb 19:200432.

10. Shi H, Han X, Jiang N, Cao Y, Alwalid O, Gu J, et al. Radiological findings from 81 patients with COVID-19 pneumonia in Wuhan, China: a descriptive study. The Lancet Infectious diseases. 2020 Feb 24.

11. Li L, Qin L, Xu Z, Yin Y, Wang X, Kong B, et al. Artificial Intelligence Distinguishes COVID-19 from Community Acquired Pneumonia on Chest CT. Radiology. 2020 Mar 19:200905.

12. Wang S, Kang B, Ma J, Zeng X, Xiao M, Guo J, et al. A deep learning algorithm using CT images to screen for Corona Virus Disease (COVID19). medRxiv. 2020.

13. Bai HX, Wang R, Xiong Z, Hsieh B, Chang K, Halsey K, et al. Al Augmentation of Radiologist Performance in Distinguishing COVID-19 from Pneumonia of Other Etiology on Chest CT. Radiology. 2020 Apr 27:201491.

14. https://www.cell.com/pb-assets/products/coronavirus/CELL_CELL-D-20-00656.pdf.

15. Dong D, Tang Z, Wang S, Hui H, Gong L, Lu Y, et al. The role of imaging in the detection and management of COVID-19: a review. IEEE reviews in biomedical engineering. 2020 Apr 27.

16. Wang S, Zha Y, Li W, Wu Q, Li X, Niu M, et al. A Fully Automatic Deep Learning System for COVID-19 Diagnostic and Prognostic Analysis. medRxiv. 2020.

17. Chen J, Wu L, Zhang J, Zhang L, Gong D, Zhao Y, et al. Deep learning-based model for detecting 2019 novel coronavirus pneumonia on highresolution computed tomography: a prospective study. medRxiv. 2020.

18. Shan F, Gao Y, Wang J, Shi W, Shi N, Han M, et al. Lung Infection Quantification of COVID-19 in CT Images with Deep Learning. arXiv preprint 2020;arXiv:2003.04655.

19. Gozes O, Frid-Adar M, Greenspan H, Browning P, Zhang H, Ji W, et al. Rapid Al Development Cycle for the Coronavirus (COVID-19) Pandemic: Initial Results for Automated Detection \& Patient Monitoring using Deep Learning CT Image Analysis. arXiv preprint. 2020;arXiv:2003.05037.

20. Dong D, Zhang F, Zhong LZ, Fang MJ, Huang CL, Yao JJ, et al. Development and validation of a novel MR imaging predictor of response to induction chemotherapy in locoregionally advanced nasopharyngeal cancer: a randomized controlled trial substudy (NCT01245959). BMC medicine. 2019 Oct 23;17(1):190.

21. Lambin P, Rios-Velazquez E, Leijenaar R, Carvalho S, van Stiphout RG, Granton P, et al. Radiomics: extracting more information from medical images using advanced feature analysis. European journal of cancer (Oxford, England : 1990). 2012 Mar;48(4):441-6.

22. Huang YQ, Liang CH, He L, Tian J, Liang CS, Chen X, et al. Development and Validation of a Radiomics Nomogram for Preoperative Prediction of Lymph Node Metastasis in Colorectal Cancer. Journal of clinical oncology : official journal of the American Society of Clinical Oncology. 2016 Jun 20;34(18):2157-64.

23. Dong D, Tang L, Li ZY, Fang MJ, Gao JB, Shan XH, et al. Development and validation of an individualized nomogram to identify occult peritoneal metastasis in patients with advanced gastric cancer. Annals of oncology : official journal of the European Society for Medical Oncology. 2019 Mar 1;30(3):431-8.

24. Chen X, Tang Y, Mo Y, Li S, Lin D, Yang Z, et al. A diagnostic model for coronavirus disease 2019 (COVID-19) based on radiological semantic and clinical features: a multi-center study. European radiology. 2020 Apr 16.

25. Qi X, Jiang Z, Yu Q, Shao C, Zhang H, Yue H, et al. Machine learning-based CT radiomics model for predicting hospital stay in patients with pneumonia associated with SARS-CoV-2 infection: A multicenter study. medRxiv. 2020.

26. Fang M, He B, Li L, Dong D, Yang X, Li C, et al. CT radiomics can help screen the coronavirus disease 2019 (COVID-19): a preliminary study. SCIENCE CHINA Information Sciences. 2020;63:172103.

27. Yu Q, Wang Y, Huang S, Liu S, Zhou Z, Zhang S, et al.

Multicenter cohort study demonstrates more consolidation in upper lungs on initial CT increases the risk of adverse clinical outcome in COVID19 patients. Theranostics. 2020;10(12):5641-8.

28. Mandell LA, Wunderink RG, Anzueto A, Bartlett JG, Campbell GD, Dean NC, et al. Infectious Diseases Society of America/American Thoracic Society consensus guidelines on the management of community-acquired pneumonia in adults. Clinical infectious diseases : an official publication of the Infectious Diseases Society of America. 2007;44 Suppl 2:S27-S72.

Page $7 / 17$ 
29. Li Z, Zhang S, Zhang J, Huang K, Wang Y, Yu Y. MVP-Net: Multi-view FPN with Position-aware Attention for Deep Universal Lesion Detection. International Conference on Medical Image Computing and Computer-Assisted Intervention. 2019; Springer.

30. Wang X, Zhang Q, Zhou Z, Liu F, Yu Y, Wang Y, et al., editors. Evaluating Multi-Class Segmentation Errors with Anatomical Priors. 2020 IEEE 17th International Symposium on Biomedical Imaging (ISBI); 2020 3-7 April 2020.

31. Breiman L. Random Forests. Machine Learning. 2001;45(1):5-32.

32. Song F, Shi N, Shan F, Zhang Z, Shen J, Lu H, et al. Emerging 2019 Novel Coronavirus (2019-nCoV) Pneumonia. Radiology. 2020;295(1):210-7.

33. Qin L, Yang Y, Cao Q, Cheng Z, Wang X, Sun Q, et al. A predictive model and scoring system combining clinical and CT characteristics for the diagnosis of COVID-19. European radiology. 2020.

\section{Tables}

Table 1. Characteristics of Patients in the training and testing Cohorts 


\begin{tabular}{|c|c|c|c|c|c|c|}
\hline \multirow[b]{2}{*}{ Characteristic } & \multicolumn{3}{|c|}{ Training cohort } & \multicolumn{3}{|c|}{ testing cohort } \\
\hline & COVID-19 & $\begin{array}{l}\text { Other types of- } \\
\text { pneumonia }\end{array}$ & $P$ value & COVID-19 & $\begin{array}{l}\text { Other types of } \\
\text { pneumonia }\end{array}$ & $\begin{array}{l}\mathrm{p} \\
\text { Value }\end{array}$ \\
\hline Patients & 52 & 60 & - & 11 & 11 & - \\
\hline Exams & 206 & 69 & - & 38 & 13 & - \\
\hline Age & $52.7 \pm 12.6$ & $41.5 \pm 15.5$ & $<0.001^{b}$ & $47.5 \pm 16.1$ & $46.9 \pm 18.4$ & $0.942^{\mathrm{b}}$ \\
\hline Gender,Male(\%) & $31(60 \%)$ & $33(55 \%)$ & $0.623^{a}$ & $5(45 \%)$ & $8(73 \%)$ & $0.387^{\mathrm{a}}$ \\
\hline Exposure history & & & $0.006^{a}$ & & & $0.375^{\mathrm{a}}$ \\
\hline $\begin{array}{l}\text { Close contact with infected } \\
\text { patients }\end{array}$ & $52(100 \%)$ & $52(87 \%)$ & & $8(73 \%)$ & $6(55 \%)$ & \\
\hline Unknown cause & $0(0 \%) 3$ & $8(13 \%)$ & & $3(27 \%)$ & $5(45 \%)$ & \\
\hline Comorbidities, No. (\%) & & & - & & & - \\
\hline Cardiovasular disease & $1(2 \%)$ & $1(2 \%)$ & $1.000^{\mathrm{a}}$ & $2(18 \%)$ & $1(9 \%)$ & $0.534^{\mathrm{a}}$ \\
\hline Diabetes & $4(8 \%)$ & $0(0 \%)$ & $0.029^{a}$ & $0(0 \%)$ & $0(0 \%)$ & - \\
\hline Hypertension, No. (\%) & $9(17 \%)$ & $4(7 \%)$ & $0.080^{\mathrm{a}}$ & $0(0 \%)$ & $1(9 \%)$ & $0.317^{\mathrm{a}}$ \\
\hline COPD & $3(6 \%)$ & $2(3 \%)$ & $0.535^{a}$ & $0(0 \%)$ & $0(0 \%)$ & - \\
\hline Chronic liver disease & $2(4 \%)$ & $1(2 \%)$ & $0.478^{a}$ & $0(0 \%)$ & $0(0 \%)$ & - \\
\hline Chronic kidney disease & $0(0 \%)$ & $0(0 \%)$ & - & $0(0 \%)$ & $1(9 \%)$ & $0.317^{\mathrm{a}}$ \\
\hline Cancer, No. (\%) & $0(0 \%)$ & $1(2 \%)$ & $0.352^{\mathrm{a}}$ & $1(9 \%)$ & $2(18 \%)$ & $0.544^{\mathrm{a}}$ \\
\hline \multicolumn{7}{|l|}{ Symptoms } \\
\hline Fever, No. (\%) & $46(88 \%)$ & $46(77 \%)$ & $0.104^{\mathrm{a}}$ & $6(55 \%)$ & $7(64 \%)$ & $0.672^{\mathrm{a}}$ \\
\hline Cough, No. (\%) & $43(83 \%)$ & $26(43 \%)$ & $<0.001^{a}$ & $6(55 \%)$ & $3(27 \%)$ & $0.387^{\mathrm{a}}$ \\
\hline Myalgia, No. (\%) & $10(19 \%)$ & $2(3 \%)$ & $0.007^{a}$ & $2(18 \%)$ & $0(0 \%)$ & $0.476^{\mathrm{a}}$ \\
\hline Fatigue, No. (\%) & $18(35 \%)$ & $2(3 \%)$ & $<0.001^{\mathrm{a}}$ & $0(0 \%)$ & $0(0 \%)$ & - \\
\hline Headache, No. (\%) & $9(17 \%)$ & $1(2 \%)$ & $0.01^{\mathrm{a}}$ & $2(18 \%)$ & $0(0 \%)$ & $0.476^{\mathrm{a}}$ \\
\hline Nausea, No. (\%) & $5(10 \%)$ & $0(0 \%)$ & $0.046^{\mathrm{a}}$ & $2(18 \%)$ & $0(0 \%)$ & $0.476^{\mathrm{a}}$ \\
\hline Diarrhea, No. (\%) & $7(13 \%)$ & $1(2 \%)$ & $0.04^{\mathrm{a}}$ & $1(9 \%)$ & $0(0 \%)$ & $1.000^{\mathrm{a}}$ \\
\hline bellyache, No. (\%) & $0(0 \%)$ & $0(0 \%)$ & - & $1(9 \%)$ & $0(0 \%)$ & $1.000^{\mathrm{a}}$ \\
\hline Dyspnea, No. (\%) & $1(2 \%)$ & $0(0 \%)$ & $0.464^{\mathrm{a}}$ & $0(0 \%)$ & $0(0 \%)$ & - \\
\hline Other symptoms, No. (\%) & $19(37 \%)$ & $14(23 \%)$ & $0.126^{a}$ & $4(36 \%)$ & $2(18 \%)$ & $0.635^{\mathrm{a}}$ \\
\hline \multicolumn{7}{|l|}{ Laboratory results } \\
\hline White blood cell count & $4.8 \pm 2.0$ & $8.2 \pm 3.5$ & $<0.001^{b}$ & $5.1 \pm 1.8$ & $9.4 \pm 3.4$ & $0.001^{\mathrm{b}}$ \\
\hline Number of neutrophils, $\times 10 \otimes / L$ & $3.2 \pm 1.9$ & $5.8 \pm 3.2$ & $<0.001^{b}$ & $3.2 \pm 1.5$ & $6.8 \pm 3.3$ & $0.003^{b}$ \\
\hline Lymphocyte count, ×10『/L & $1.2 \pm 0.8$ & $1.6 \pm 1.1$ & $0.032^{b}$ & $1.4 \pm 0.5$ & $1.7 \pm 1.0$ & $0.473^{b}$ \\
\hline Hemoglobin & $134.1 \pm 25.2$ & $138.7 \pm 17.6$ & $0.267^{\mathrm{b}}$ & $128.3 \pm 19.8$ & $139.7 \pm 21.3$ & $0.206^{b}$ \\
\hline Platelet count, $\times 10 \rrbracket / L$ & $175.8 \pm 60.3$ & $225.6 \pm 62.0$ & $<0.001^{b}$ & $215.6 \pm 70.7$ & $230.1 \pm 89.0$ & $0.678^{b}$ \\
\hline
\end{tabular}


$\mathrm{p}^{\mathrm{a}}$ : chi-square test, $\mathrm{p}^{\mathrm{b}}:$ Student's t test. $\mathrm{p}^{\mathrm{c}}:$ Kruskal-Wallis $\mathrm{H}$ test

Table 2. The performance of CT radiomics models in training, inter-validation and testing cohorts.

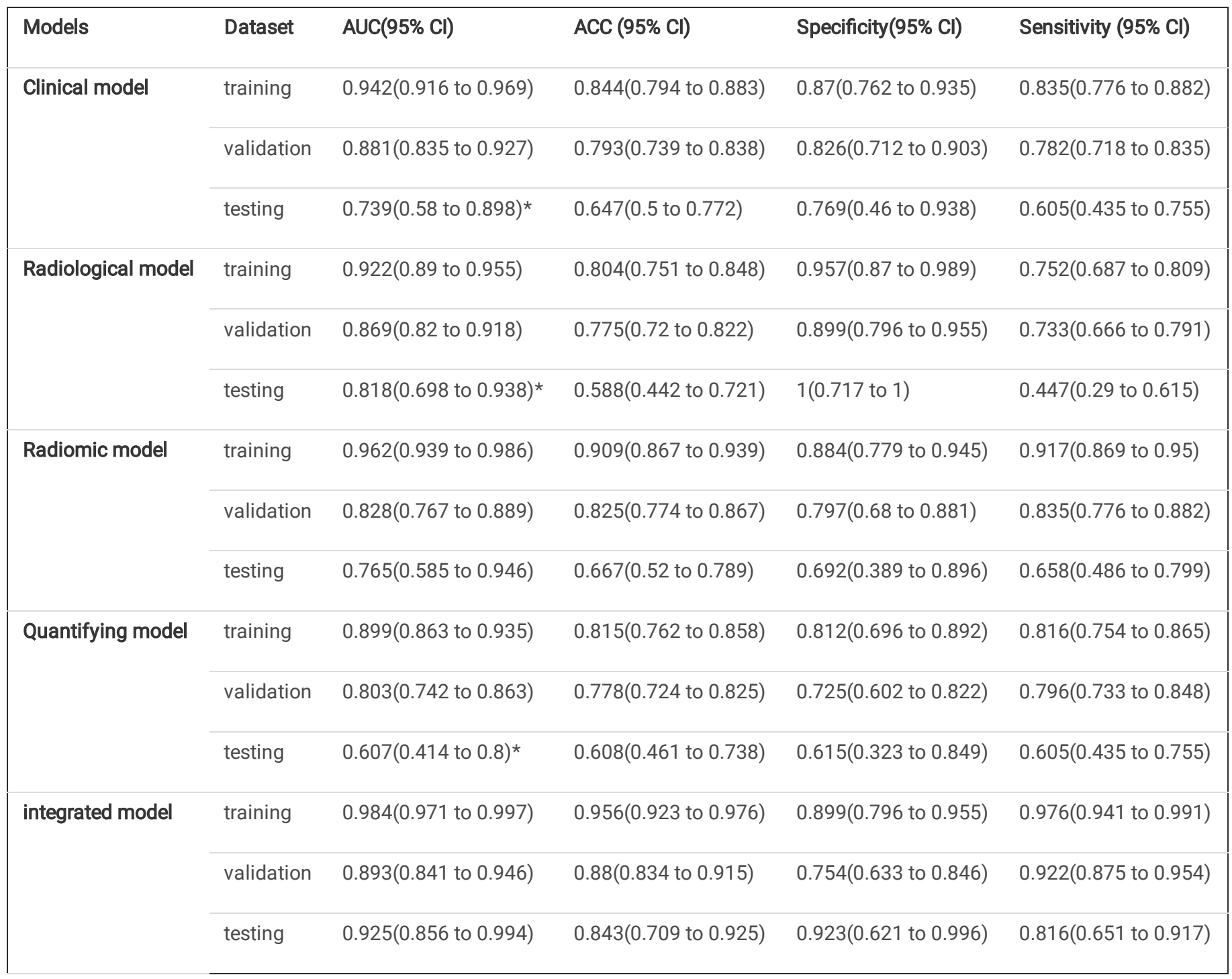

* DeLong test showed significant different $(\mathrm{p}<0.05)$ between the model with integrated model on the testing cohort. Cl confidence interval

\section{Figures}




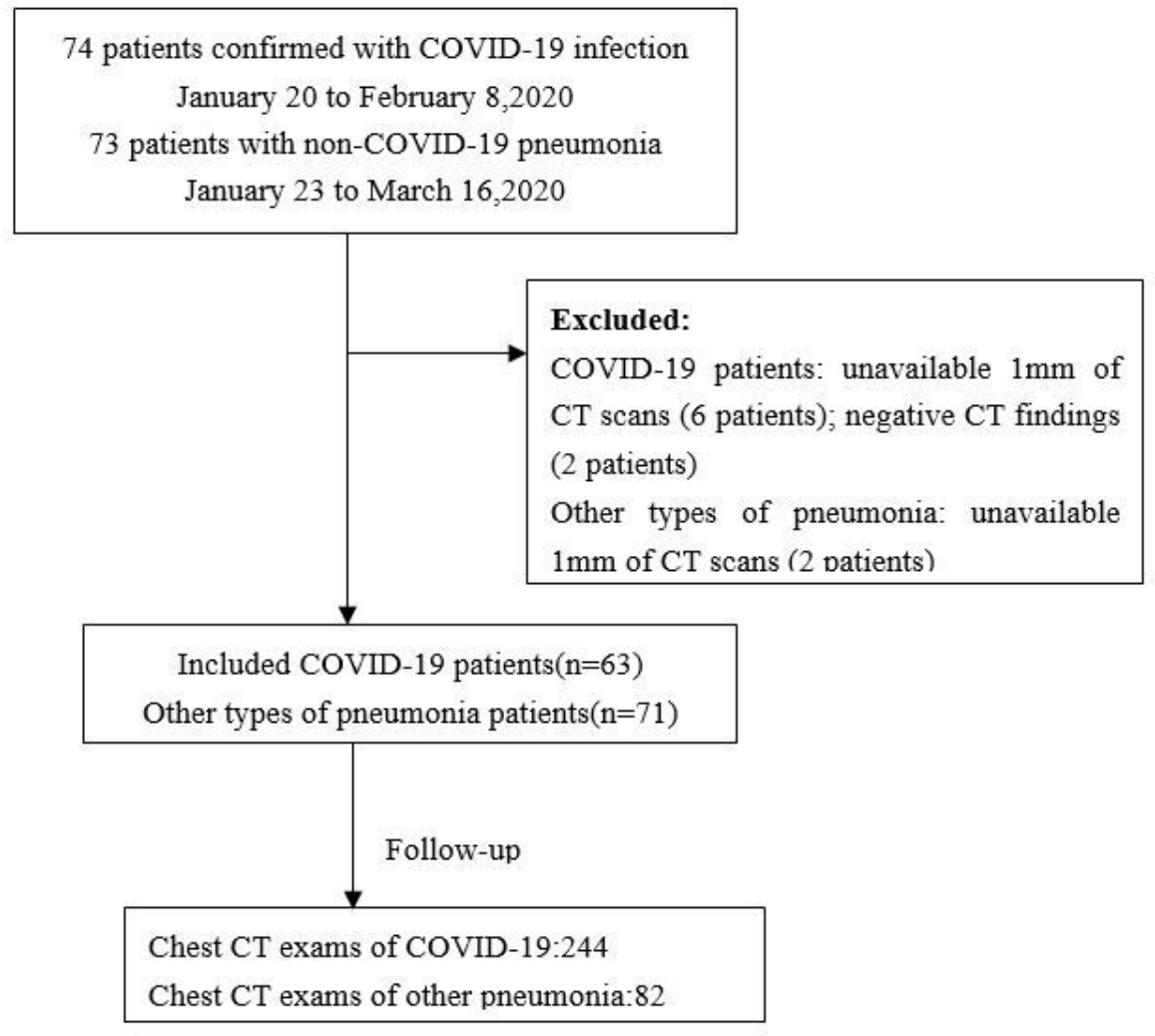

\section{Figure 1}

Flowchart of this study. 


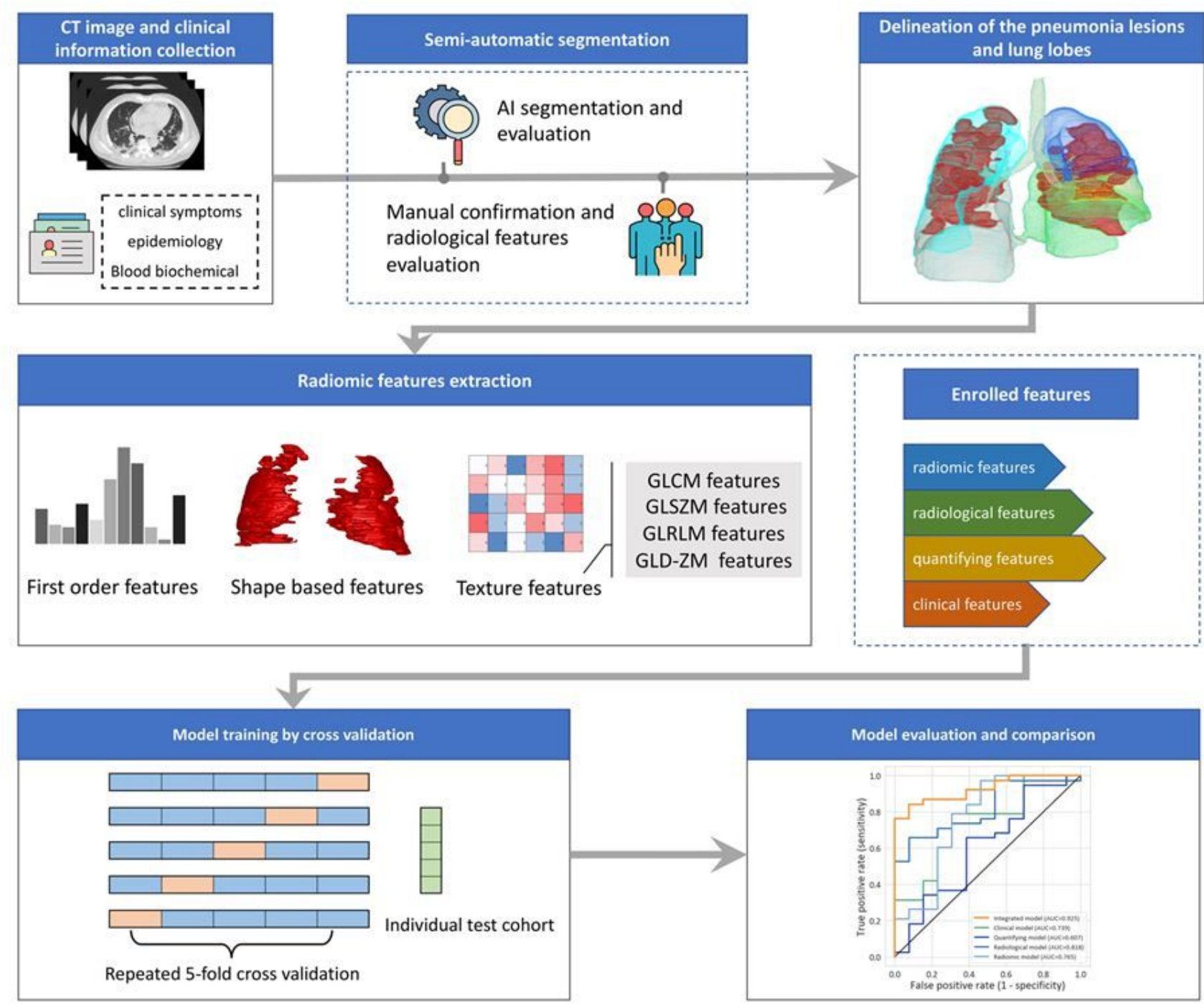

Figure 2

The workflow of our study, consisting of data collection, semi-automatic ROI segmentation and radiological features annotation, radiomic and quantifying features extraction, model building and evaluation. 

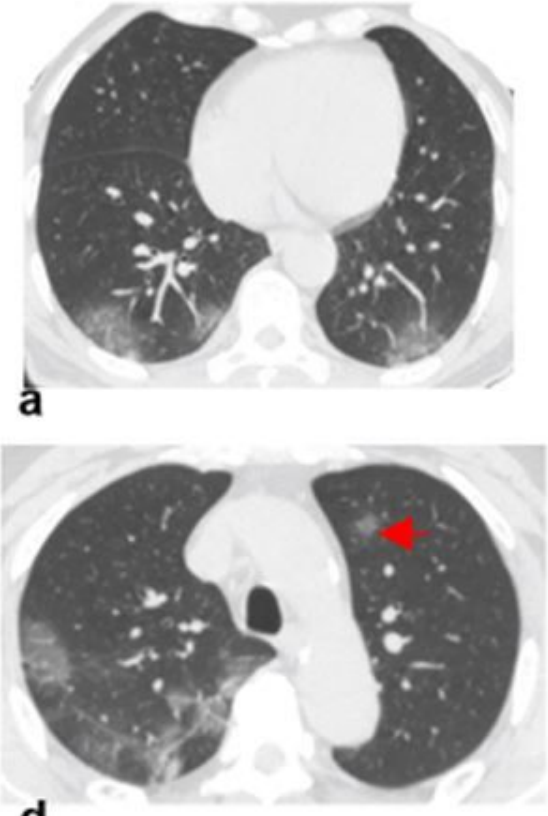

d

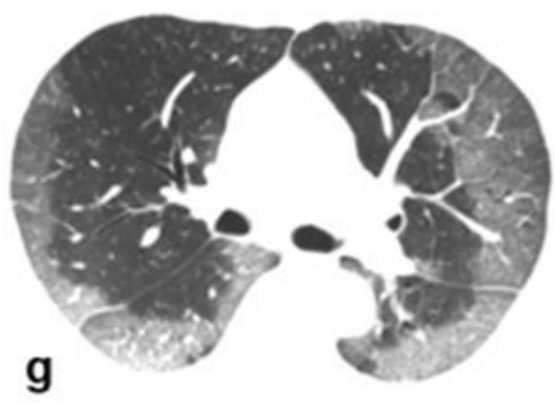

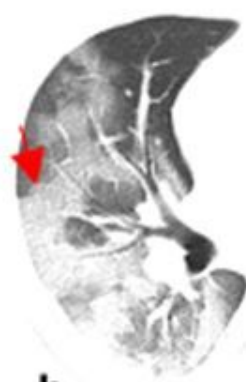

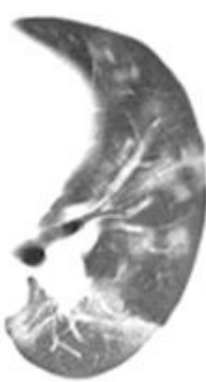

b

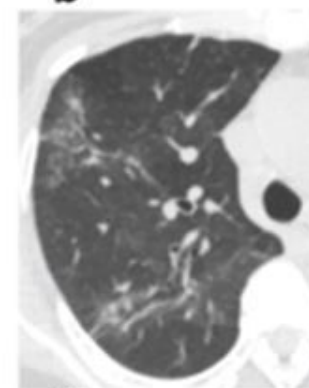

e
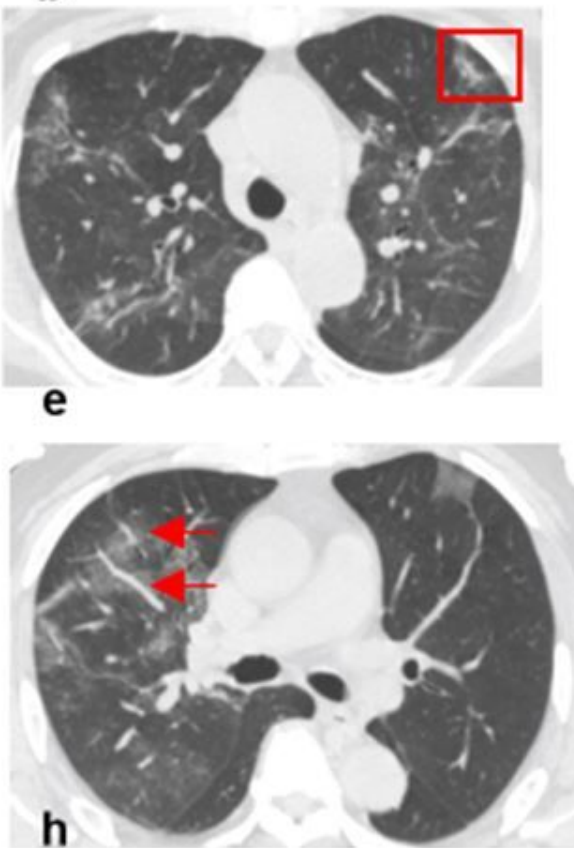

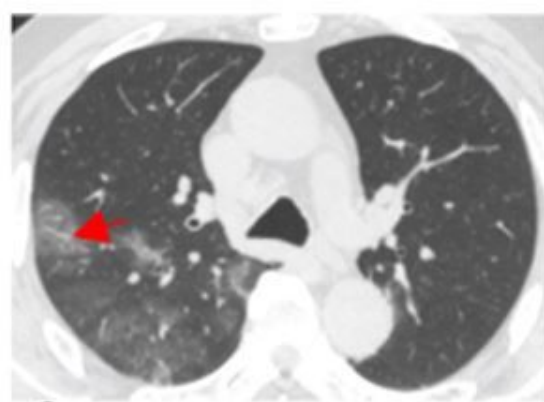

C

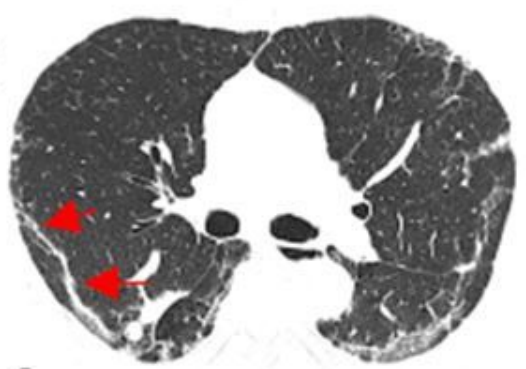

f

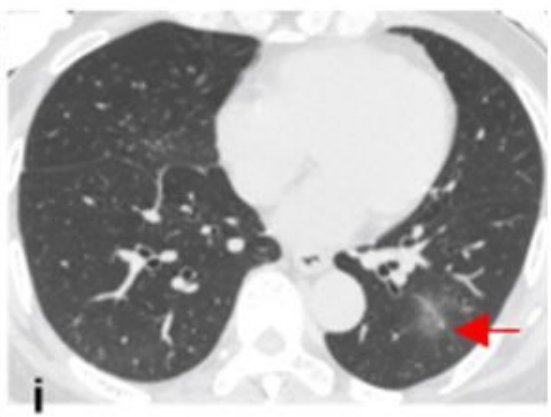

\section{Figure 3}

Typical radiological characteristics of CT manifestations. a-i demonstrated the typical radiological characteristics of ground grass opacity, crazy paving pattern, halo sign, reversed halo sign, vascular perforating in the lesion, subpleural line, subpleural distribution, bronchovascular bundle distribution and pulmonary band distribution, respectively. 


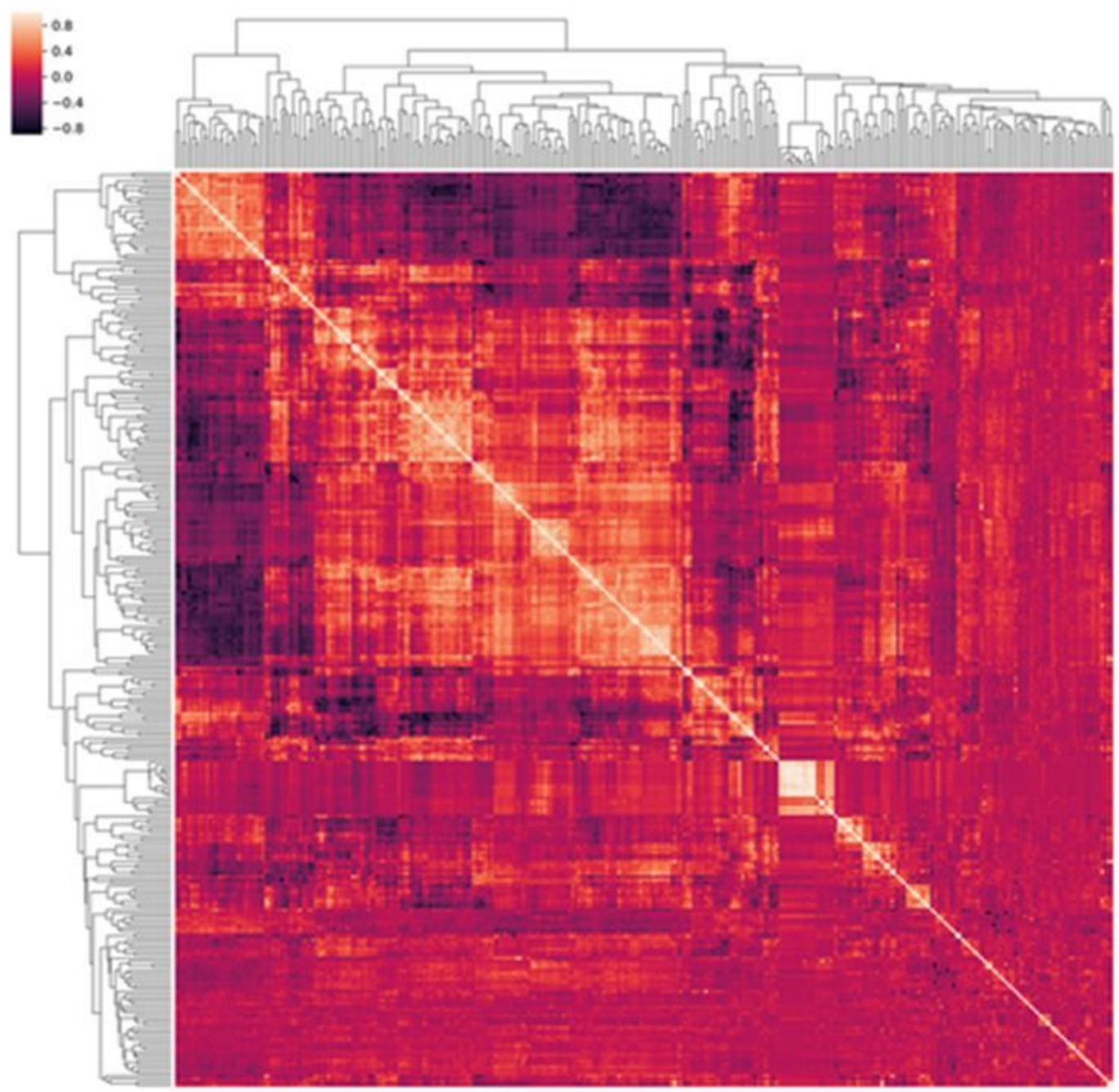

Figure 4

Clustered heatmap. Feature correlation matrix of radiomic features were represented as a hierarchically clustered heatmap. 


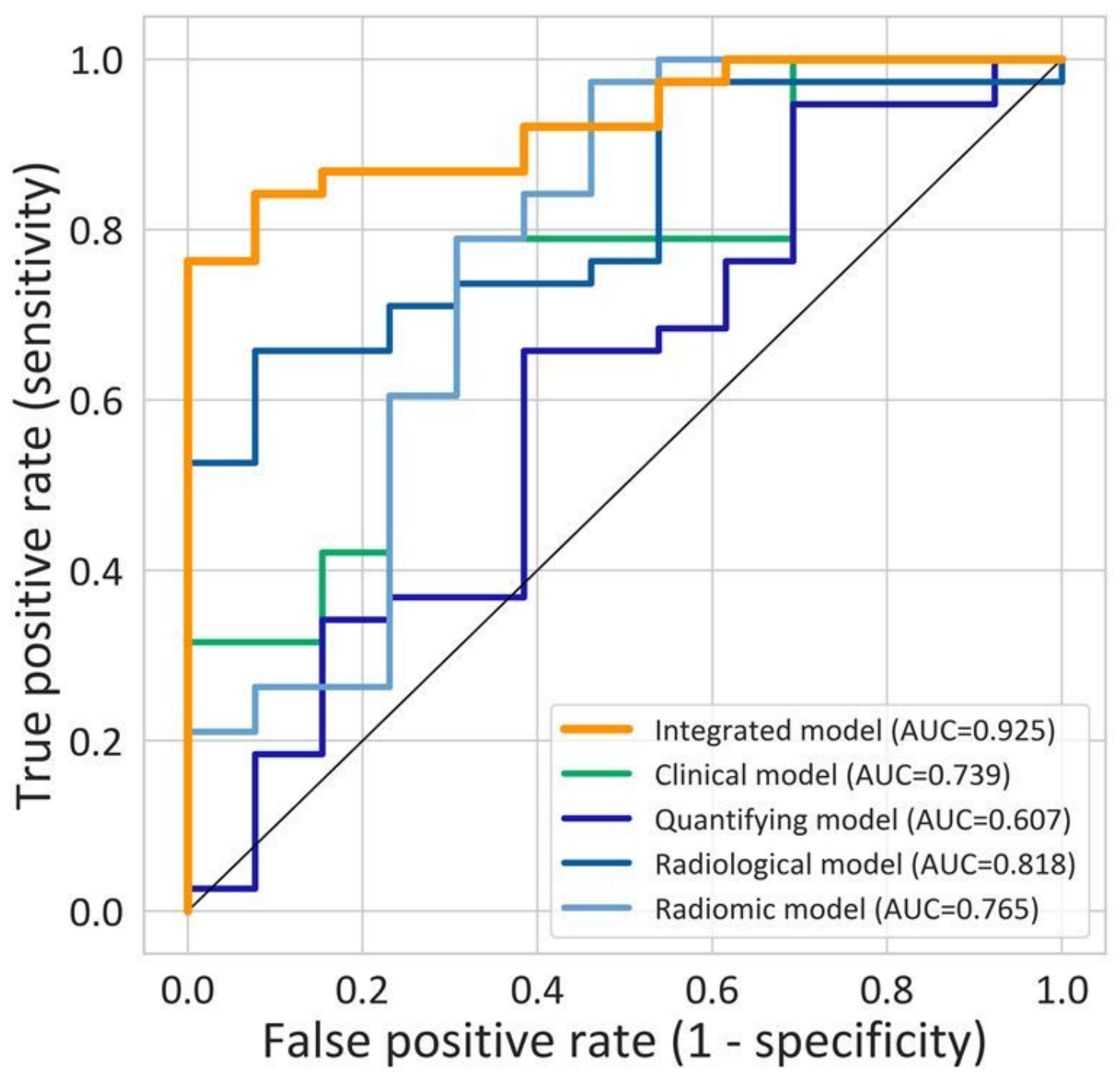

Figure 5

The ROC of the integrated model, clinical model, quantifying model, radiological model, and radiomic model on the testing cohort. 


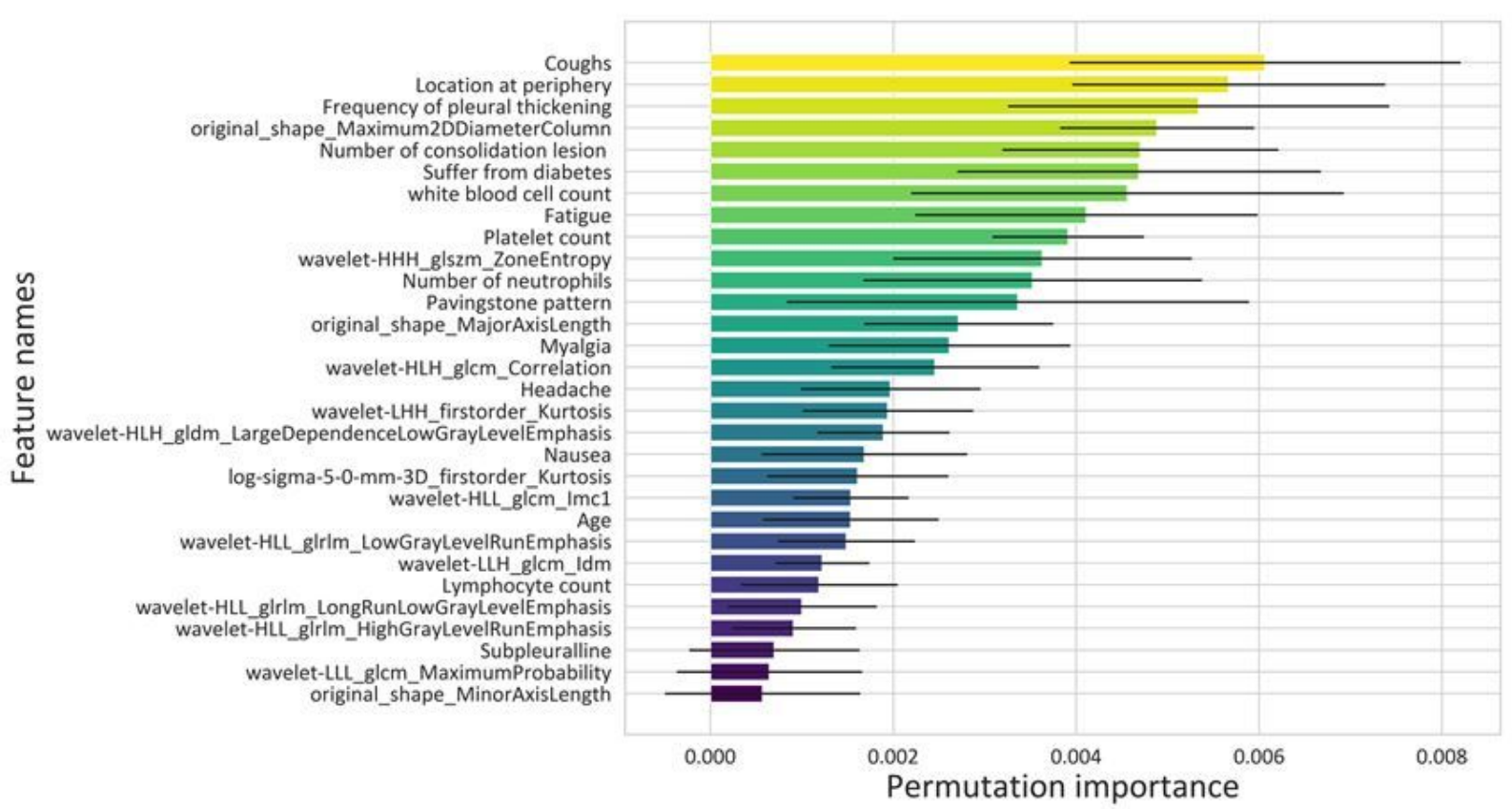

Figure 6

The permutation feature importance of the integrated model.

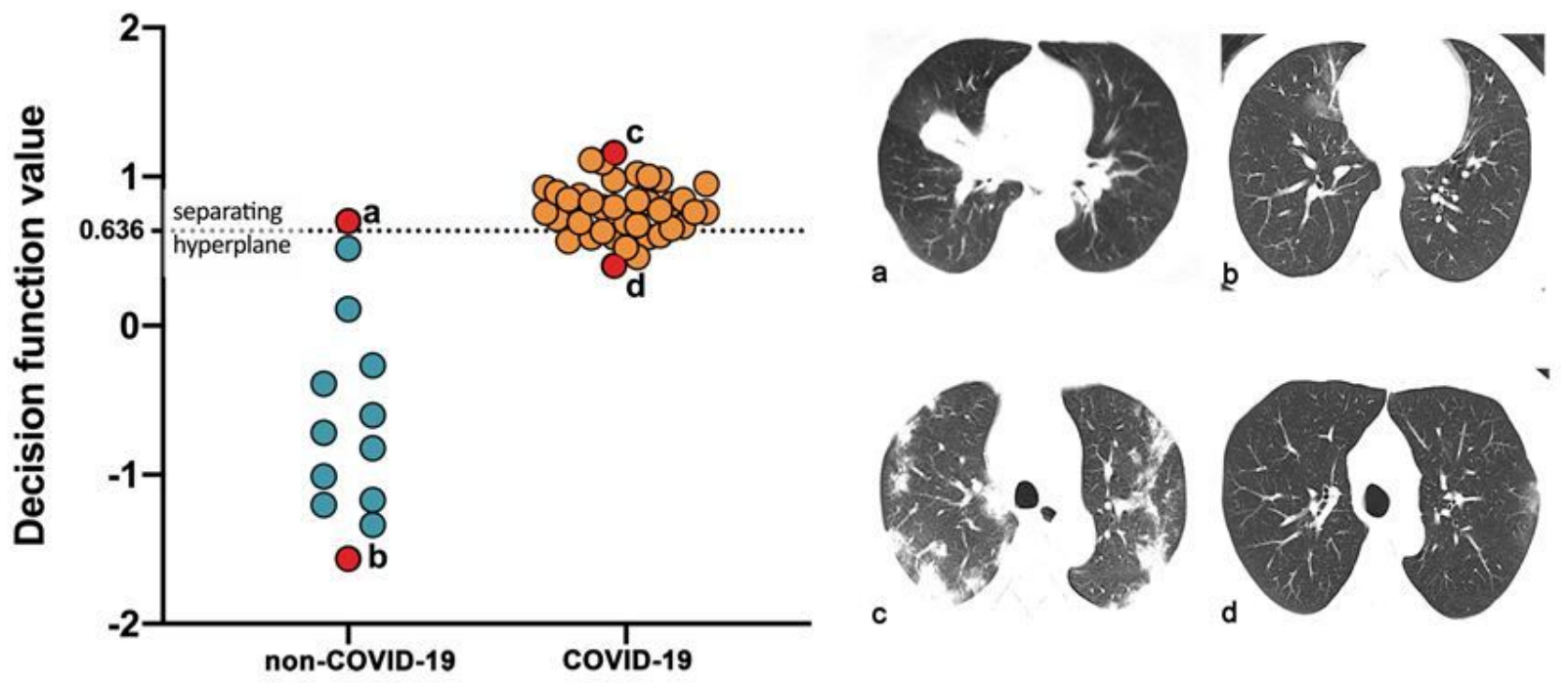

Figure 7

The decision function value distribution of the patients with non-COVID-19 pneumonia and COVID-19 in the test cohort were shown. Each point indicated a patient in the test cohort, the non-COVID-19 point below the adjusted separating hyperplane line and the COVID-19 point above the line were separated correctly. The images of the 4 typical patients were shown. (a) A patient with non-COVID-19 that misclassified as COVID-19. (b) A patient with non-COVID-19 that were correctly identified. (c) A patient with COVID-19 that were correctly identified. (d)A patient with COVID-19 that misclassified as non-COVID-19.

\section{Supplementary Files}

This is a list of supplementary files associated with this preprint. Click to download. 
- Supplementarymaterial.docx

Page 17/17 